\title{
Intravoxel incoherent motion diffusion-weighted imaging in differentiating uterine fibroid from focal adenomyosis: initial results
}

\author{
Tao Tian', Guo-Fu Zhang ${ }^{2}$, He Zhang ${ }^{2 *}$ and Hui Liu ${ }^{3}$
}

*Correspondence:

dr.zhanghe@yahoo.com

${ }^{2}$ Department of Radiology,

Obstetrics and Gynecology

Hospital, Fudan University,

No. 419 Fang xie Road,

Shanghai 200011, People's

Republic of China

Full list of author information

is available at the end of the article

\begin{abstract}
To evaluate the performance of intravoxel incoherent motion (IVIM)-diffusion-weighted imaging (DWI) in differentiating uterine fibroids from focal adenomyosises. Twentyfive uterine fibroids and 21 focal adenomyosises prospectively underwent IVIM-DWI examination prior to surgery. Four parameters including apparent diffusion coefficient total values (ADCtot), true diffusion coefficient $(D)$, pseudodiffusion coefficient $\left(D^{*}\right)$ and perfusion fraction (f) derived from IVIM-DWI images were separately calculated and compared across four groups. There was a statistically significant difference in IVIMderived $f$ parameter between fibroid and focal adenomyosis $(p=0.01)$ and control group ( $p=0.02$ ). Uterine fibroids gave higher coefficient of variation (CV) of all IVIMderived parameters than focal adenomyosises. IVIM-DWI could improve the sensitivity and specificity of detecting focal adenomyosis to 100 and $92.6 \%$, respectively. IVIM- $f$ parameter could be potentially used to better distinguish uterine fibroid from focal adenomyosis. The higher CV of IVIM-derived parameters with acceptable range is often observed in the diseased group.
\end{abstract}

Keywords: Intravoxel incoherent motion, Diffusion-weighted imaging, Uterine fibroid, Focal adenomyosis

\section{Background}

Adenomyosis was firstly defined as benign invasion of endometrium in the myometrium, producing a diffusely enlarged uterus by Bird et al. in 1972(Bird and ManaloEstrella 1972; Garcia and Isaacson 2011). It commonly affects premenopausal women and is associated with clinical manifestations similar to uterine fibroids (Azziz 1989), which are the most common tumor of the reproductive tract in women (Bulman et al. 2012). It is necessary to accurately differentiate uterine fibroid from focal adenomyosis owing to various therapeutic approaches.

With the advantages of the superb soft tissue resolution and no radiation, magnetic resonance imaging (MRI) has been widely performed to image pelvic diseases in clinical unit, especially for indeterminate masses on ultrasound (Sala et al. 2013). In recent studies, diffusion weighted imaging (DWI) has been used to distinguish malignant

(c) 2016 Tian et al. This article is distributed under the terms of the Creative Commons Attribution 4.0 International License (http:// creativecommons.org/licenses/by/4.0/), which permits unrestricted use, distribution, and reproduction in any medium, provided you give appropriate credit to the original author(s) and the source, provide a link to the Creative Commons license, and indicate if changes were made. 
tumors from benign gynaecological diseases with promising results (Zhang et al. 2012; Thomassin-Naggara et al. 2013). It has been recognized that the calculated ADC value from lower $b$ value images is more sensitive to capillary perfusion, representing motion of intravascular water protons within imaging voxels (Koh and Orton 2011; Takahara and Kwee 2012). Thus, IVIM approach proposed by Le Bihan et al. (1986), by using biexponential analysis, could integrate both tissue perfusion and diffusion effects in DWI images. Three parameters derived by IVIM, named as $D$ (true diffusion coefficient), $D^{*}$ (pseudodiffusion coefficient) and $f$ (perfusion fraction), are quantitative indexes used to reflect diffusion and perfusion changes in various tissues, i.e., head, liver, pancreas, colon, uterus and prostate (Lemke et al. 2009; Shinmoto et al. 2012; Sumi et al. 2012; Chiaradia et al. 2014; Doblas et al. 2013; Bisdas et al. 2013; Lee et al. 2014a).

To date, application of IVIM to image female pelvic diseases is still limited. The purpose of this study was to determine whether IVIM models could be explored to discriminate uterine fibroid from focal adenomyosis.

\section{Results and discussion}

Finally, a total of 56 consecutive qualified subjects were recruited into the studied group, including 21 participants (25-52 years of age; average age, $37.9 \pm 7.3$ ) in focal adenomyosis group, 25 (28-69 years of age; average age, 44.4 \pm 10.6$)$ in uterine fibroid group and 10 with normal uterine structure as control group (24-69 years of age; average age, $40.9 \pm 10.5$ ). Others (23 endometrium cancer, 40 cervical cancer, 1 uterine sarcoma and 10 with unavailable patients' consent, 6 with claustrophobia and 7 with no final histological diagnosis) were excluded. The average SMR for both fibroids and focal adenomyosis was described in additional file 1: Figure S1. The details of SNR at varying b values DWI images were summarized in additional file 2: Figure S2.

The mean values of IVIM parameters for uterine fibroid (Fig. 1) were: ADCtot $=1.31 \pm 0.43\left(\times 10^{-3} \mathrm{~mm}^{2} / \mathrm{s}\right), D=1.12 \pm 0.43\left(\times 10^{-3} \mathrm{~mm}^{2} / \mathrm{s}\right), D^{*}=15.9 \pm 5.0$ $\left(\times 10^{-3} \mathrm{~mm}^{2} / \mathrm{s}\right), f(\%)=10.5 \pm 6.3$, respectively; for focal adenomyosis (Fig. 2) were: ADCtot $=1.09 \pm 0.14\left(\times 10^{-3} \mathrm{~mm}^{2} / \mathrm{s}\right), D=0.95 \pm 0.13\left(\times 10^{-3} \mathrm{~mm}^{2} / \mathrm{s}\right), D^{*}=16.8 \pm 5.0$ $\left(\times 10^{-3} \mathrm{~mm}^{2} / \mathrm{s}\right), f(\%)=15.7 \pm 3.6$, respectively; for control group were: ADCtot $=1.24 \pm 0.19\left(\times 10^{-3} \mathrm{~mm}^{2} / \mathrm{s}\right), D=1.18 \pm 0.21\left(\times 10^{-3} \mathrm{~mm}^{2} / \mathrm{s}\right), D^{*}=18.6 \pm 3.8$ $\left(\times 10^{-3} \mathrm{~mm}^{2} / \mathrm{s}\right), f(\%)=16.6 \pm 8.0$, respectively (Table 1$)$.

The statistically significant differences were only observed in $f$ parameter between fibroid and focal adenomyosis $(p=0.01)$ and control group $(p=0.02)$ (Figs. 3, 4). The detailed significant differences of IVIM parameters at statistical level within three groups were listed in Table 2. Regarding the repeatability of the IVIM-based parameters, the CVs of ADCtot, $D, D^{*}$ and $f$ between uterine fibroid and focal adenomyosis group were $0.31,0.25,0.17,0.44$ and $0.14,0.19,0.38,0.20$, respectively. The CVs of the IVIM model parameters in uterine fibroid were relatively higher than focal adenomyosis group, while much higher than the control group (Table 3). The Bland-Altman plots demonstrated satisfactory results without any outliers outside the mean \pm 1.96 SD boundaries in all cases, indicating a good agreement in both inter-observer reliability and intra-observer reproducibility (Fig. 5). On $\mathrm{T}_{1} \mathrm{WI}$, both fibroid and focal adenomyosis appeared intermediate signals (similar with myometrium). On $\mathrm{T}_{2} \mathrm{WI}$, most fibroids appeared as low signals (14/21), seven cases showed iso/hyper signals (similar with endometrium); for focal 


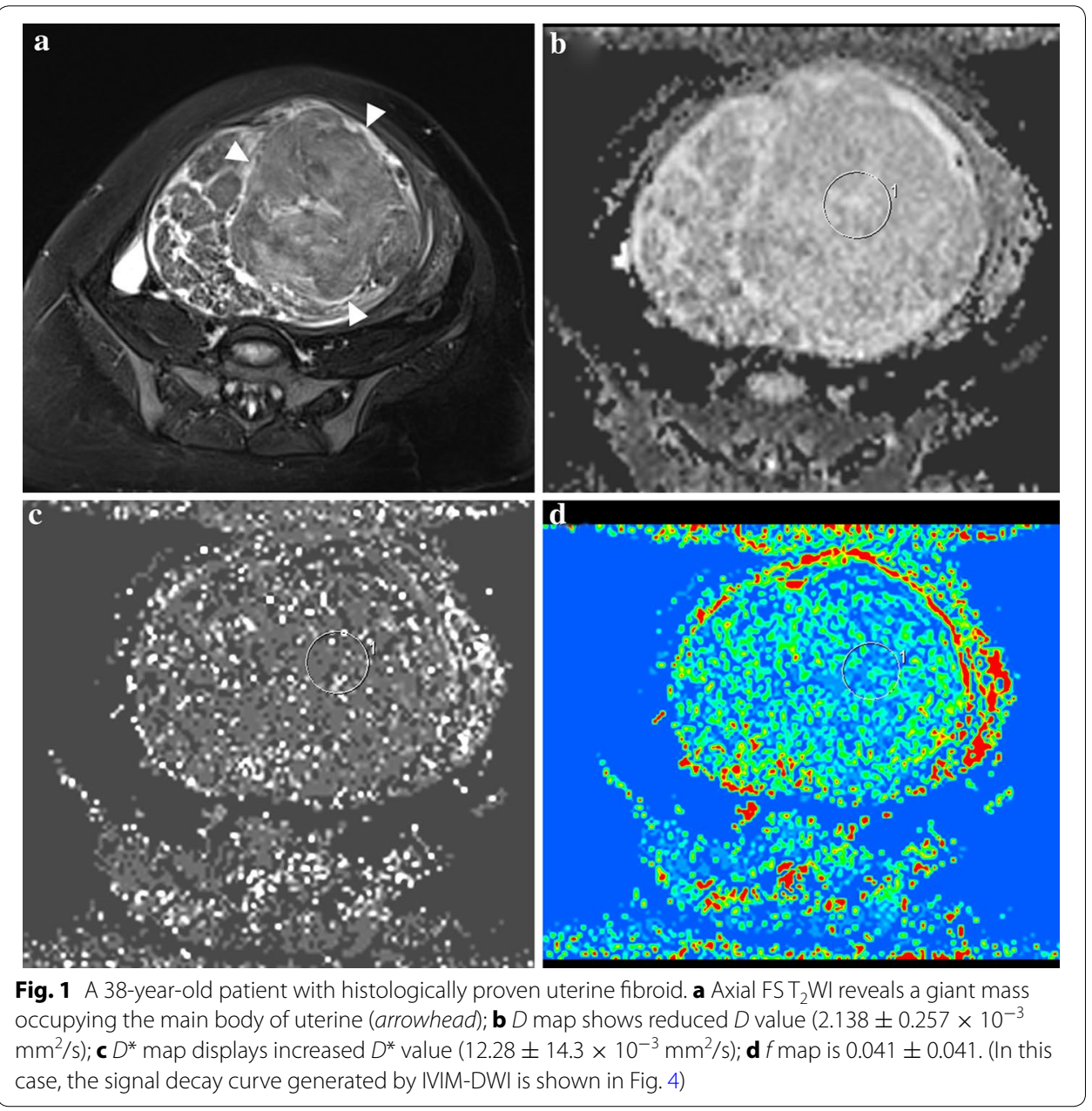

adenomyosis, all lesions in the studied samples were iso/hypo signals on $\mathrm{T}_{1} \mathrm{WI}$ and iso/ hyper signals on $\mathrm{T}_{2} \mathrm{WI}$. Overall, combining with IVIM-DWI information, the sensitivity and specificity of MRI for detecting focal adenomyosis were 100 and $92.6 \%$, respectively, which was higher than only with conventional MRI reading session (Table 4).

Both fibroid and adenomyosis are the most common benign condition of the uterus in women of reproductive age and often coexist with similar clinical complains (Jha et al. (2014). On MRI, these two etiologies could be easily differentiated based on imaging signs and specific lesion characters (Takeuchi and Matsuzaki 2011). Sometimes it is difficult to discriminate focal adenomyosis or small adenomoyoma from fibroids (Matsumoto et al. 2013). Considering various treatments for each etiology, accurate diagnosis is still needed prior to aggressive treatments. Here, we reported our preliminary experiences with IVIM-MRI approach in differentiation between uterine fibroid and focal adenomyosis in our institution. Our data showed that IVIM- $f$ was a more robust index than IVIM- $D$ and IVIM- $D^{*}$ parameter to discriminate uterine fibroid from focal adenomyosis with no overlap $(p=0.01)$.

DWI-MRI is a functional imaging technique that is now widely applied in categorizing suspected lesions, staging malignancies and monitoring therapeutic effects (Sala et al. 

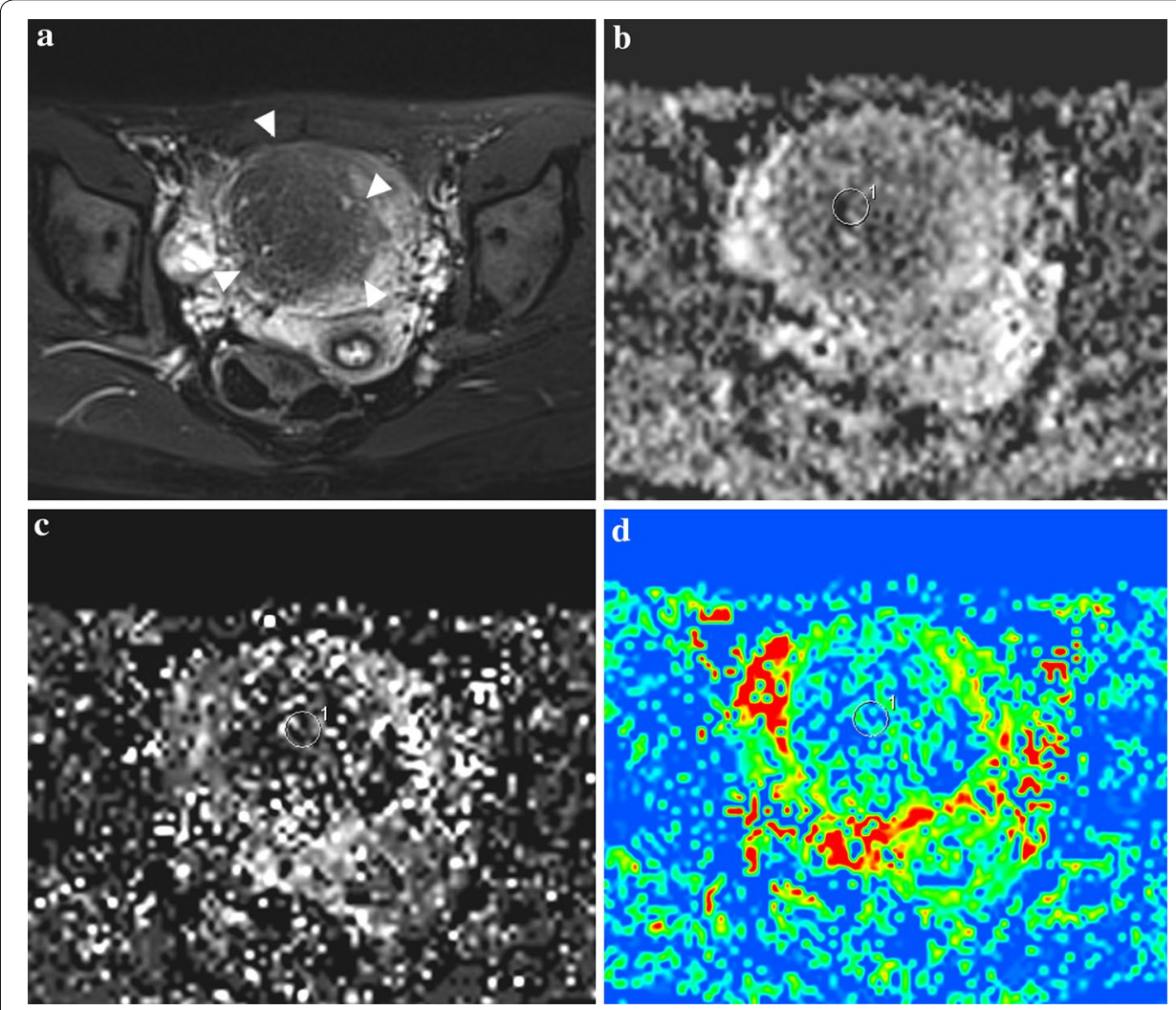

Fig. 2 A 25-year-old patient with histologically proven focal adenomyosis. a Axial FS $T_{2} W I$ reveals a oval mass with main hypointensity signal occupying the myometrium and the junctional zone (arrowhead); $\mathbf{b} D$ map shows the mass is homogeneously isointensity signal with the $D$ value of $0.515 \pm 0.358 \times 10^{-3} \mathrm{~mm}^{2} / \mathrm{s}$; $\mathbf{c} D^{*}$ map displays the $D^{*}$ value is $18.8 \pm 19.9 \times 10^{-3} \mathrm{~mm}^{2} / \mathrm{s}$; $\mathbf{d}$ the $f$ map is $0.114 \pm 0.101$. (In this case, the signal decay curve generated by IVIM-DWI is shown in Fig. 4)

Table 1 Comparison of IVIM parameters (mean \pm standard deviation) between uterine fibroid, focal adenomyosis and control group

\begin{tabular}{|c|c|c|c|c|c|}
\hline Group & $\mathrm{N}$ & $\begin{array}{l}\text { ADCtot } \\
\left(10^{-3} \mathrm{~mm}^{2} / \mathrm{s}\right)\end{array}$ & $\begin{array}{l}D \\
\left(10^{-3} \mathrm{~mm}^{2} / \mathrm{s}\right)\end{array}$ & $\begin{array}{l}D^{*} \\
\left(10^{-3} \mathrm{~mm}^{2} / \mathrm{s}\right)\end{array}$ & $f(\%)$ \\
\hline Uterine fibroid & 21 & $1.31 \pm 0.4$ & $1.12 \pm 0.4$ & $15.9 \pm 5.5$ & $10.5 \pm 6.3$ \\
\hline Focal adenomyosis & 25 & $1.09 \pm 0.1$ & $0.95 \pm 0.1$ & $16.8 \pm 5.0$ & $15.7 \pm 3.6$ \\
\hline Control group & 10 & $1.24 \pm 0.2$ & $1.18 \pm 0.2$ & $18.6 \pm 3.8$ & $16.6 \pm 8.0$ \\
\hline
\end{tabular}

2013; Zhang et al. 2012; Stamatopoulos et al. 2012; Lee et al. 2014b; Zhang et al. 2014). By using multiple $b$ values, IVIM-DWI could potentially incorporate both perfusion and diffusion information to describe the tissue signal attenuation with mathematical model fitting (Koh and Orton 2011; Takahara and Kwee 2012; Le Bihan et al. 1986). In contrast to perfusion parameters derived from other techniques (i.e., dynamic contrast enhanced imaging), owing to the advantages of free-contrast and shorter acquisition time, IVIMDWI has gained increasing attractions in clinically relevant application (Lemke et al. 2009; Sumi et al. 2012; Chiaradia et al. 2014; Doblas et al. 2013). Several recent studies 

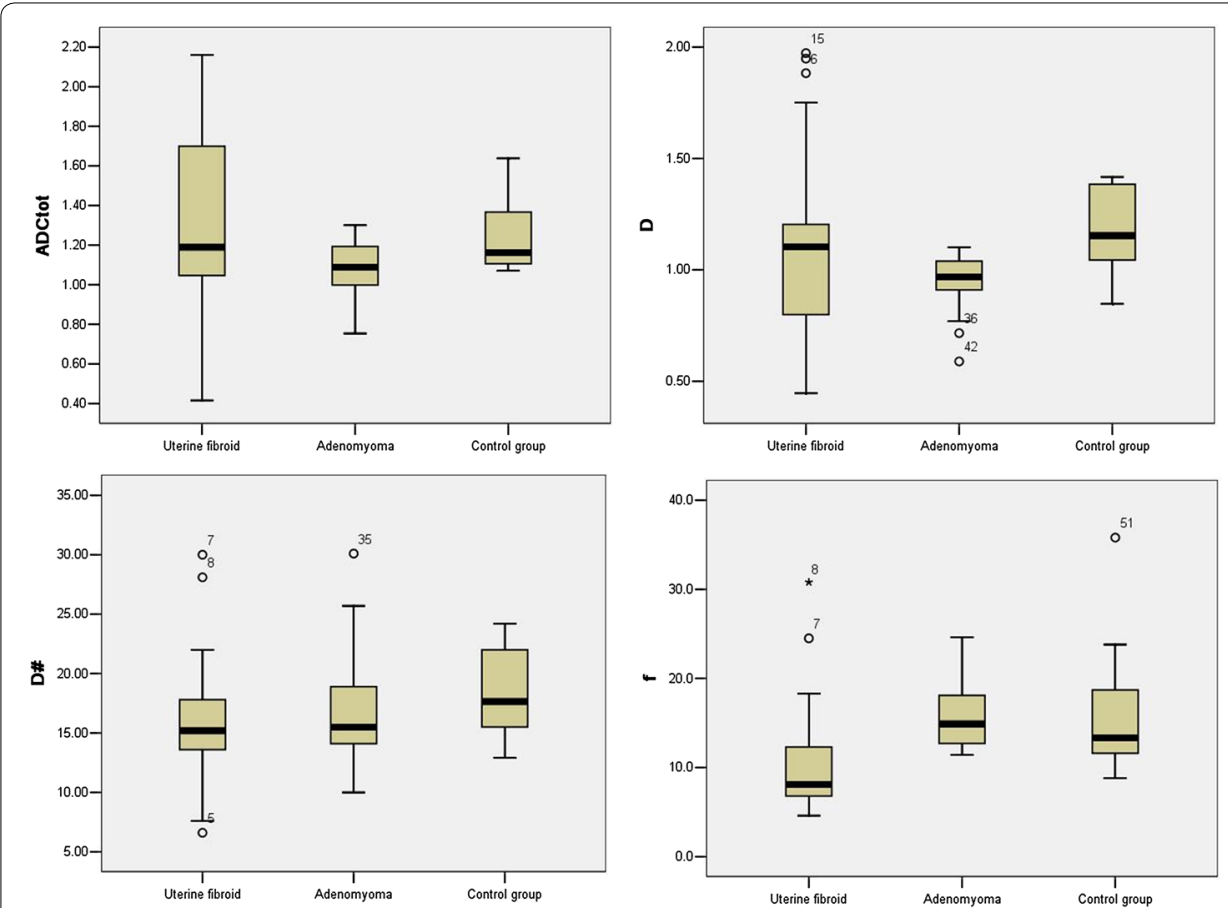

Fig. 3 Box plots (top and bottom of boxes represent $25-75 \%$ of the data values; line in box represents median value; circles represents the outliers; asterisk represents extreme cases) of ADCtot $\left(10^{-3} \mathrm{~mm}^{2} / \mathrm{s}\right), D$ $\left(10^{-3} \mathrm{~mm}^{2} / \mathrm{s}\right), D^{*}\left(10^{-3} \mathrm{~mm}^{2} / \mathrm{s}\right)$ and $f(\%)$ in uterine fibroid, focal adenomyosis and control group. Note, $f$ in uterine fibroid is significantly lower than focal adenomyosis $(p=0.01)$ and control group ( $p=0.02)$

with focus on IVIM-derived parameters in various tissues characterization have been published (Liu et al. 2013; Lu et al. 2013; Sumi and Nakamura 2014).

There are mainly four mathematical models including the monoexponential model, the stretched exponential model, the kurtosis model and the biexponential model to quantify DWI signal decay (Jambor et al. 2014; Merisaari and Jambor 2014). Among them, a monoexponential fit model is the simplest mathematical model to define signal decay with more robust parameter than the other three models (Takahara and Kwee 2012). In one study, the authors declared that the parameters calculated with monoexponential, kurtosis, and stretched-exponential models had better reliability and repeatability of the fitted parameters than the biexponential model (Merisaari and Jambor 2014). Our study corroborated this point that the CV of IVIM-derived parameters $\left(f, D, D^{*}\right)$ from the biexponential model was relatively large and may be more sensitive to noise.

In one study, Yang et al. investigated the value of DWI at 3.0-Tesla MR unit in the differentiating uterine adenomyosis from uterine fibroids, suggesting uterine adenomyosis demonstrated significantly higher mean ADC values than uterine leiomyoma (Yang et al. 2011). In our study, there was no difference in ADCtot values derived from IVIM images between uterine fibroid and focal adenomyosis $(p=0.072)$. Of note, in the studied fibroid group, IVIM-derived parameters have much larger variation compared with the other two groups. Inhomogeneous signals on $\mathrm{T}_{2} \mathrm{WI}(7 / 21)$ were more often observed in fibroid group, indicating some likely degeneration which may influence the final calculation. 


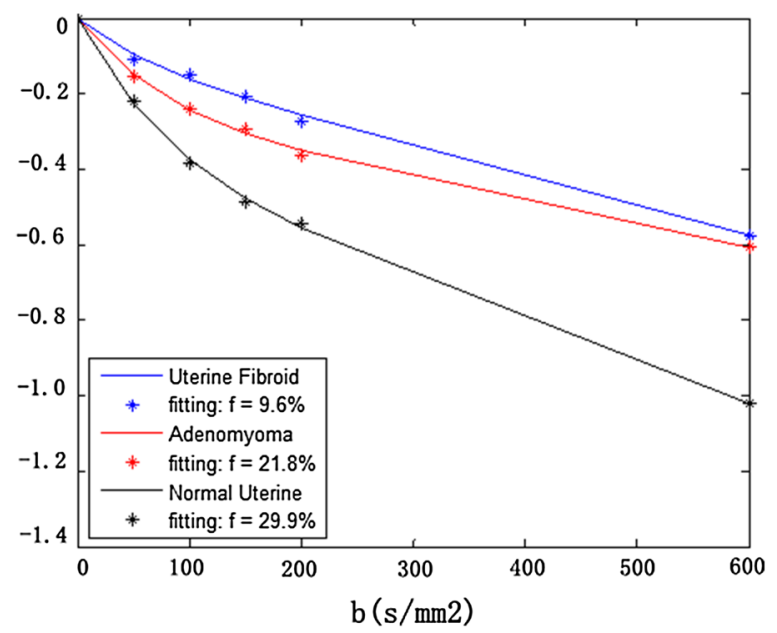

Fig. 4 Biexponential fit of the signal decay in according with the varying $b$ values in three selected samples. Blue line represents uterine fibroid (Fig. 1); red line represents focal adenomyosis (Fig. 2); black line represents the normal uterine. Note, IVIM-f in uterine fibroid significantly lows than that in both focal adenomyosis and control group

Table 2 The statistically significant difference ( $p$ value) of IVIM parameters within three groups

\begin{tabular}{|c|c|c|c|c|}
\hline & ADCtot & $D\left(10^{-3} \mathrm{~mm}^{2} / \mathrm{s}\right)$ & $D^{*}\left(10^{-3} \mathrm{~mm}^{2} / \mathrm{s}\right)$ & $f(\%)$ \\
\hline Uterine fibroid and focal adenomyosis & 0.072 & 0.146 & 0.836 & 0.010 \\
\hline Uteine fibroid and control group & 0.829 & 0.890 & 0.347 & 0.020 \\
\hline Focal adenomyosis and control group & 0.445 & 0.143 & 0.626 & 0.930 \\
\hline
\end{tabular}

Table 3 Coefficient variations of IVIM parameters measurements in 11 subjects within three groups

\begin{tabular}{llll}
\hline Parameters & Uterine fibroid & Focal adenomyosis & Control group \\
\hline ADCtot & 0.31 & 0.14 & 0.004 \\
$D\left(10^{-3} \mathrm{~mm}^{2} / \mathrm{s}\right)$ & 0.25 & 0.19 & 0.004 \\
$D^{*}\left(10^{-3} \mathrm{~mm}^{2} / \mathrm{s}\right)$ & 0.17 & 0.38 & 0.08 \\
$f(\%)$ & 0.44 & 0.20 & 0.16 \\
\hline
\end{tabular}

Our results demonstrated that IVIM- $f$ parameter (reflecting tissue microcapillary perfusion) could be a potential indicator in differentiating fibroid from focal adenomyosis (10.5 vs. $15.7 \%, p=0.01)$. These data well correlated with the histological results that proliferative ectopic endometrial tissues in the myometrium contains plenty of capillary vessels, increasing blood flow volume in the whole lesion. Further, IVIM images could also aid radiologists to improve their diagnostic performance in discriminating fibroid from adenomyosis before invasive procedure.

Inter-examination reproducibility is an important estimation of the reliability of IVIM as a clinically useful discriminator. In this study, $D$ is much more reliable (19-25\%) compared with $D^{*}(17-38 \%)$ and $f(20-44 \%)$, consistent with published results from another 

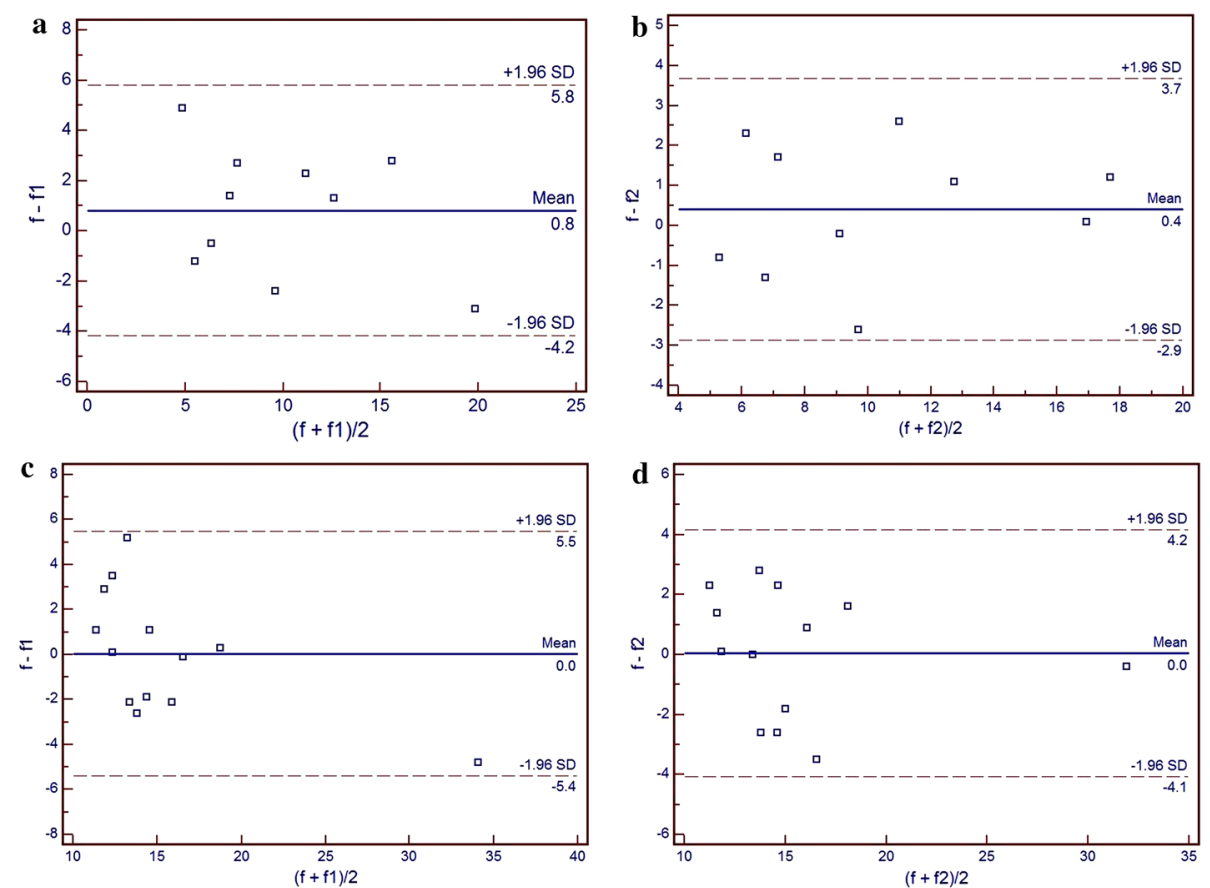

Fig. 5 Bland-Altman plots estimate the interobserver reliability $(\mathbf{a}, \mathbf{c})$ and intraobserver repeatability $(\mathbf{c}$, d) of the IVIM-f parameters in uterine fibroid $(\mathbf{a}, \mathbf{b})$ and focal adenomyosis $(\mathbf{c}, \mathbf{d})$. The differences in the $f$ values between the first and the second measurements ( $y$-axis) are plotted against the averages of them ( $x$-axis), with mean difference and $95 \%$ limits of agreement indicated

Table 4 Diagnostic performance according to two kinds of MRI protocols

\begin{tabular}{lccccc}
\hline Protocol & SEN (\%) & SPE (\%) & PPV (\%) & NPV (\%) & ACC (\%) \\
\hline Conventional MRI & $90.0(18 / 20)$ & $88.5(23 / 26)$ & $85.7(18 / 21)$ & $92.0(23 / 25)$ & $89.1(41 / 46)$ \\
& $(68.3-98.8)$ & $(69.9-97.6)$ & $(63.7-97.9)$ & $(74.0-99.0)$ & $(75.6-95.9)$ \\
Conventional MRI & $100.0(19 / 19)$ & $92.6(25 / 27)$ & $90.5(21 / 23)$ & $100.0(25 / 25)$ & $95.7(44 / 46)$ \\
plus IVIM & $(82.4-100.0)$ & $(75.7-99.0)$ & $(69.6-98.8)$ & $(86.3-100.0)$ & $(85.5-98.8)$ \\
\hline
\end{tabular}

Numbers in parentheses are the data used to calculate the percentages. Numbers in brackets are $95 \%$ confidence intervals; conventional MRI includes T1 wi/T2wi/contrast-enhanced MRI

study (Lai et al. 2013). In general, these variations were acceptable, especially regarding the much lower $\mathrm{CV}$ of IVIM-derived indexes in the control group.

There were several limitations of this study. Firstly, the IVIM processing software we used here is not commercially standardized till now; the purpose of these acquisition protocols mainly apply for scientific research. Secondly, we selected six $b$ values to acquire IVIM-DWI data, which was different with other studies. Theoretically, the choice of much lower $b$ values may more accurately reflect perfusion sensitive signal attenuation (Koh and Orton 2011; Takahara and Kwee 2012). However, free-breathing technique was used to accommodate multiple $b$ values in this study, thus, patients movements are unavoidable when increase the acquisition time length; SNR variations which may also be accordingly elevated, resulting in inaccurate signal measurements at multiple $b$ values images. The total acquisition time of $3.5 \mathrm{~min}$ in this study was acceptable for all patients and the signal decay fitting line can also be roughly modeled (Fig. 4). Thirdly, 
ROIs were manually drawn and individually calculated on a case-by-case basis, and lack of standardization may effect on the final results. Finally, high-field MR unit (3 Tesla) has been gradually introduced into the clinical market. It is also needed to determine whether or not there is any difference in application of IVIM in uterine lesions between 1.5T and 3T MR unit.

\section{Conclusions}

In summary, IVIM- $f$ can be used as a quantitiative parameter to better differentiate uterine fibroid from focal adenomyosis. The higher CVs of IVIM-derived parameters with acceptable range are more often observed in the disease group than the control group.

\section{Methods}

\section{Study subjects}

This study was approved by our institutional review board. Patients or qualifying family members provided their written informed consent before participation. From March 2013 to June 2013, 143 consecutive patients with clinically suspected pelvic disease prospectively underwent MRI and IVIM examination. Laparotomy or laparoscopic surgery was performed to confirm the etiology of uterine diseases. The time interval between MRI and surgery was less than 1 month. Inclusion criteria were: (1) newly suspected uterine diseases; (2) no previous treatment history. Exclusion criteria were: (1) contraindication for MRI examinations; (2) uncooperative patients or unavailable of patient's consents.

\section{Image acquisition}

MR imaging was performed using a 1.5-T MR system (Magnetom Avanto, Siemens, Erlangen, Germany) with a phased-array coil. The routine MRI protocols used for the assessment of pelvic masses included the axial turbo spin-echo (TSE) $\mathrm{T}_{1}$-weighted imaging $\left(\mathrm{T}_{1} \mathrm{WI}\right.$, repetition time/echo time $\left.(\mathrm{TR} / \mathrm{TE})=550 / 10 \mathrm{~ms}\right)$, sagittal TSE $\mathrm{T}_{2}$-weighted imaging $\left(\mathrm{T}_{2} \mathrm{WI}, \mathrm{TR} / \mathrm{TE}=4000 / 83 \mathrm{~ms}\right)$ and axial/sagittal TSE fat-suppressed $\mathrm{T}_{2} \mathrm{WI}$ $\left(\mathrm{FS} \mathrm{T}_{2} \mathrm{WI}, \mathrm{TR} / \mathrm{TE}=8000 / 83 \mathrm{~ms}\right.$ ). Diffusion-weighted imaging (DWI) using an echoplanar imaging two-dimensional (EP2D) sequence in free-breath performed in the axial plane with parallel acquisition technique (GRAPPA acceleration factor of two) by using six $b$ values $\left(0,50,100,150,200\right.$ and $\left.600 \mathrm{~s} / \mathrm{mm}^{2}\right)$. The details of acquisition parameters were as follows: $\mathrm{TR}=4000 \mathrm{~ms}, \mathrm{TE}=78 \mathrm{~ms}$, field of view $(\mathrm{FOV})=280 \mathrm{~mm}$, slice thickness $=5 \mathrm{~mm}$, bandwidth $=1726 \mathrm{~Hz} /$ Pixel, fat suppression with spectral pre saturation attenuated inversion recovery (SPAIR) technique. Average ADC map was automatically generated. The image resolution generated from IVIM yielded an approximate voxel size of $2.0 \times 2.0 \times 5.0 \mathrm{~mm}$ with a total examination time of three and a half minutes.

\section{DW-MRI data analysis}

The IVIM model is described by the equation below, where $D$ and $D^{*}$ are the diffusion parameters related with molecular diffusion and with the incoherent microcirculation respectively, $\mathrm{S}$ is the mean signal intensity and $f$ is perfusion fraction, i.e., the fraction of the pseudo-diffusion (or perfusion) correlated with microcirculation:

$$
\frac{S(b)}{S_{0}}=(1-f) \exp (-b * D)+f * \exp \left(-b *\left(D+D^{*}\right)\right)
$$


where $S_{\mathrm{i}}$ is the signal at $b=\mathrm{b}_{\mathrm{i}}, S_{0}$ is the baseline signal, where $b=0 ; D$ is the slow diffusion decay associated with extravascular water molecules' motion; $D^{*}$ is the fast diffusion decay associated with the intravascular water molecules' motion; and $f$ is the fraction perfusion compartment in the two compartments.

A work in progress post-processing program is used to fit the above IVIM bi-exponential model to generate three parametric images $\left(D, D^{*}\right.$ and $\left.f\right)$ using two segment method, where an initial estimation of $D$ using a reduced set of $b$-values larger than a predetermined value (in our case, $b=200$ is used.) and then using the resulting $D$ as a fix parameter to fit the missing parameters similar to what was described in (Luciani et al. 2008). In addition, we estimated the ADC of the mono exponential signal decay model:

$$
S_{i}=S_{0} \exp \left(-b_{i} A D C\right)
$$

where $\mathrm{S}_{\mathrm{i}}, \mathrm{b}_{\mathrm{i}}$, and $\mathrm{S}_{0}$ are as defined above. The total ADC value (ADCtot) was then measured by using the entire range of $b$-value images on IVIM-map.

\section{Image data analysis}

Firstly, all MRI image raw data were reviewed by two readers (H.Z., T.T.) blind to the final pathological results; the final conclusion was made with consensus reading. Four IVIM-derived parameters (ADCtot, $D, D^{*}$ and $f$ ) were separately measured in two sessions (with 3-month interval) for evaluating the reproducibility of data interpretation. ADCs were measured manually on commercially available post-processing workstation (Leonardo, Siemens, Germany) by one reader (H.Z.). The signal-muscle ratio (SMR) for each lesion at both $\mathrm{T}_{1} \mathrm{WI}$ and $\mathrm{T}_{2} \mathrm{WI}$ sequence and the signal-noise ratio (SNR) of DWI images at varying $b$ values were calculated by the same reader (H.Z.). Regions of interest (ROI) with average circle area from 180 to $220 \mathrm{~mm}^{2}$ was placed into the mostly solid part of each lesion in both fibroid and focal adenomyosis group. For multiple lesions in one subject, we chose the largest one as the targeted lesion for the further evaluation.

\section{Statistical analysis}

Numerical variables were expressed as the mean $\pm \mathrm{SD}$. The factor analysis within a set of measured variables across each parameter was validated by Tukey's test. The repeatability of the IVIM results was tested by the CV; A Bland-Altman analysis was employed to analyze the agreement between the two measurements. The receiver operating characteristic (ROC) curve was calculated for each IVIM parameter in differentiating fibroid from adenomyosis. The diagnostic performance of MRI based on two series of protocols (conventional MRI and conventional MRI plus IVIM) were calculated as accuracy (ACC), sensitivity (SEN), specificity (SPE), positive predictive values (PPV), and negative predictive values (NPV), expressing as percentages [95\% confidence interval (CI)]. A p value less than 0.05 was considered statistically significant. SPSS (version 13.0, SPSS Inc., Chicago, USA) and MedCalc (version9.2.1.0, MedCalc Sofware, Ostend, Belgium) were used to perform statistical. 


\section{Additional files}

Additional file 1: Figure S1. Illustrating case of signal-noise-ratio calculation at various b values DWI images. A 29-year-old female with focal adenomyoma in the posterior wall of uterine. One reviewer (H.Z.) placed the ROI with average area $2.54 \mathrm{~cm}^{2}$ in the center of lesion. Similarly, on the same series of pictures, $\mathrm{ROI}_{2}$ with an average area of $2.80 \mathrm{~cm}^{2}$ indicating the noise signals was also placed on the background. The SNR values in ten cases in each group were calculated. To minimum the operator bias, only one experienced operator did the whole procedure. Additional file 1: Figure S2. SNR values at various $b$ values in three groups. The final SNR values for each group were $28.2 \pm 9.2$ at $b=0,32.6 \pm 4.3$ at $b=50,29.9 \pm 0.8$ at $b=100,27.5 \pm 2.1$ at $b=150,25.9 \pm 4.3$ at $b=200$, $17.0 \pm 1.0$ at $b=600$ for myoma and $32.1 \pm 2.0$ at $b=0,49.3 \pm 2.0$ at $b=50,48.9 \pm 5.9$ at $b=100,47.9 \pm 1.9$ at $b=150,41.4 \pm 2.9$ at $b=200,30.7 \pm 3.3$ at $b=600$ for adenomyoma and $45.8 \pm 2.2$ at $b=0,63.3 \pm 2.5$ at $b=50,52.5 \pm 4.4$ at $b=100,46.7 \pm 5.2$ at $b=150,45.8 \pm 3.5$ at $b=200,27.6 \pm 3.3$ at $b=600$ for control group, respectively.

\section{Abbreviations}

IVIM: intravoxel incoherent motion; DWI: diffusion weighted imaging; ADC: apparent diffusion coefficient; CV: coefficient of variation; MRI: magnetic resonance imaging.

\section{Author's contributions}

$\mathrm{HZ}$ and GZ designed of the whole study; HZhang TT and HL performed the experiments and analyzed the data; $H Z$ revised and wrote the manuscript finally. All authors read and approved the final manuscript.

\section{Author details}

${ }^{1}$ Department of Radiology, Shanghai No. 9 Hospital, School of Medicine, Shanghai Jiaotong University, Shanghai, People's Republic of China. ${ }^{2}$ Department of Radiology, Obstetrics and Gynecology Hospital, Fudan University, No. 419 Fang xie Road, Shanghai 200011, People's Republic of China. ${ }^{3}$ Collaboration Scientist, MR Business Group, Healthcare Sector, Siemens Medical Solutions Ltd., Shanghai, People's Republic of China.

\section{Acknowledgements}

This work was supported by Shanghai Natural Science Funding grant (Project no. 14ZR1404400).

\section{Competing interests}

The authors declare that they have no competing interests. The authors of this manuscript declare no relationships with any companies.

Received: 8 November 2015 Accepted: 17 December 2015

Published online: 04 January 2016

\section{References}

Azziz R (1989) Adenomyosis: current perspectives. Obstet Gynecol Clin North Am 16:221-235

Bird CMT, Manalo-Estrella P (1972) The elusive adenomyosis of the uterus revisited. Am J Obstet Gynecol 112:583-593 Bisdas S, Koh T, Roder C, Braun C, Schittenhelm J, Ernemann U, Klose U (2013) Intravoxel incoherent motion diffusionweighted $\mathrm{mr}$ imaging of gliomas: feasibility of the method and initial results. Neuroradiology 10:1189-1196 Bulman JC, Ascher SM, Spies JB (2012) Current concepts in uterine fibroid embolization. Radiographics 32:1735-1750 Chiaradia M, Baranes L, Van Nhieu JT, Vignaud A, Laurent A, Decaens T, Charles-Nelson A, Brugières P, Katsahian S, Djabbari M, Deux J-F, Sobhani I, Karoui M, Rahmouni A, Luciani A (2014) Intravoxel incoherent motion (IVIM) MR imaging of colorectal liver metastases: are we only looking at tumor necrosis? J Magn Reson Imaging 39(2):317-325. doi:10.1002/jmri.24172

Doblas S, Wagner M, Leitao HS, Daire J-L, Sinkus R, Vilgrain V, Van Beers BE (2013) Determination of malignancy and characterization of hepatic tumor type with diffusion-weighted magnetic resonance imaging: comparison of apparent diffusion coefficient and intravoxel incoherent motion-derived measurements. Invest Radiol 48(10):722-728

Garcia L, Isaacson K (2011) Adenomyosis: review of the literature. J Minim Invasive Gynecol 18:428-437

Jambor I, Merisaari H, Taimen P, Boström P, Minn H, Pesola M, Aronen HJ (2014) Evaluation of different mathematical models for diffusion-weighted imaging of normal prostate and prostate cancer using high b-values: a repeatability study. Magn Reson Med 73:1988-1998. doi:10.1002/mrm.25323 (Epub ahead of print)

Jha R, Zanello P, Ascher S, Rajan S (2014) Diffusion-weighted imaging (DWI) of adenomyosis and fibroids of the uterus. Abdom Imaging 39(3):562-569

Koh DMCD, Orton MR (2011) Intravoxel incoherent motion in body diffusion-weighted MRI: reality and challenges. Am J Roentgenol 196:1351-1361

Lai V, Li X, Lee V, Lam K, Chan Q, Khong P (2013) Intravoxel incoherent motion mr imaging: comparison of diffusion and perfusion characteristics between nasopharyngeal carcinoma and post-chemoradiation fibrosis. Eur Radiol 23(10):2793-2801

Le Bihan DBE, Lallemand D, Grenier P, Cabanis E, Laval-Jeantet M (1986) MR imaging of intra-voxel incoherent motions: application to diffusion and perfusion in neurologic disorders. Radiology 161:401-407

Lee EY, Yu X, Chu MM, Ngan HY, Siu SW, Soong IS, Chan Q, Khong PL (2014a) Perfusion and diffusion characteristics of cervical cancer based on intraxovel incoherent motion MR imaging-a pilot study. Eur Radiol 24(7):1506-1513 
Lee H-J, Rha SY, Chung YE, Shim HS, Kim YJ, Hur J, Hong YJ, Choi BW (2014b) Tumor perfusion-related parameter of diffusion-weighted magnetic resonance imaging: correlation with histological microvessel density. Magn Reson Med 71(4):1554-1558

Lemke A, Laun FB, Klau M, Re TJ, Simon D, Delorme S, Schad LR, Stieltjes B (2009) Differentiation of pancreas carcinoma from healthy pancreatic tissue using multiple b-values: comparison of apparent diffusion coefficient and intravoxel incoherent motion derived parameters. Invest Radiol 44(769-775):7. doi:10.1097/RLI.1090b1013e3181 b62271

Liu C, Liang C, Liu Z, Zhang S, Huang B (2013) Intravoxel incoherent motion (IVIM) in evaluation of breast lesions: comparison with conventional DWI. Eur J Radiol:S0720-0048X(0713)00405-00401

Lu Y, Jansen JFA, Stambuk HE, Gupta G, Lee N, Gonen M, Moreira A, Mazaheri Y, Patel SG, Deasy JO, Shah JP, Shukla-Dave A (2013) Comparing primary tumors and metastatic nodes in head and neck cancer using intravoxel incoherent motion imaging: a preliminary experience. J Comput Assist Tomogr 37(346-352):3. doi:10.1097/RCT.1090b1013e31 $8282 \mathrm{~d} 318935$

Luciani A, Vignaud A, Cavet M, Van Tran Nhieu J, Mallat A, Ruel L, Laurent A, Deux J-F, Brugieres P, Rahmouni A (2008) Liver cirrhosis: intravoxel incoherent motion mr imaging—pilot study. Radiology 249:891-899

Matsumoto T, Hiura M, Baba T, Ishiko O, Shiozawa T, Yaegashi N, Kobayashi H, Yoshikawa H, Kawamura N, Kaku T (2013) Clinical management of atypical polypoid focal adenomyosis of the uterus. A clinicopathological review of 29 cases. Gynecol Oncol 129:54-57

Merisaari H, Jambor I (2014) Optimization of b-value distribution for four mathematical models of prostate cancer diffusion-weighted imaging using b values up to $2000 \mathrm{~s} / \mathrm{mm}^{2}$ : simulation and repeatability study. Magn Reson Med 73:1954-1969. doi:10.1002/mrm.25310 (Epub ahead of print)

Sala E, Rockall AG, Freeman SJ, Mitchell DG, Reinhold C (2013) The added role of mr imaging in treatment stratification of patients with gynecologic malignancies: what the radiologist needs to know. Radiology 266:717-740

Shinmoto HTC, Soga S, Shiomi E, Yoshihara N, Kaji T, Mulkern RV (2012) An intravoxel incoherent motion diffusionweighted imaging study of prostate cancer. Am J Roentgenol 199:W496-W500

Stamatopoulos CP, Mikos T, Grimbizis GF, Dimitriadis AS, Efstratiou I, Stamatopoulos P, Tarlatzis BC (2012) Value of magnetic resonance imaging in diagnosis of adenomyosis and myomas of the uterus. J Minim Invasive Gynecol 19:620-626

Sumi M, Nakamura T (2014) Head and neck tumours: combined mri assessment based on ivim and tic analyses for the differentiation of tumors of different histological types. Eur Radiol 24(1):223-231

Sumi M, Van Cauteren M, Sumi T, Obara M, Ichikawa Y, Nakamura T (2012) Salivary gland tumors: use of intravoxel incoherent motion mr imaging for assessment of diffusion and perfusion for the differentiation of benign from malignant tumors. Radiology 263:770-777

Takahara T, Kwee TC (2012) Low b-value diffusion-weighted imaging: emerging applications in the body. J Magn Reson Imaging 35:1266-1273

Takeuchi M, Matsuzaki K (2011) Adenomyosis: usual and unusual imaging manifestations, pitfalls, and problem-solving mr imaging techniques. Radiographics 31:99-115

Thomassin-Naggara I, Dechoux S, Bonneau C, Morel A, Rouzier R, Carette M-F, Daraï E, Bazot M (2013) How to differentiate benign from malignant myometrial tumours using mr imaging. Eur Radiol 23:2306-2314

Yang Q, Zhang LH, Su J, Liu J (2011) The utility of diffusion-weighted mr imaging in differentiation of uterine adenomyosis and leiomyoma. Eur J Radiol 79:e47-e51

Zhang H, Zhang G-F, He Z-Y, Li Z-Y, Zhu M, Zhang G-X (2012) Evaluation of primary adnexal masses by 3T MRI: categorization with conventional mr imaging and diffusion-weighted imaging. J Ovarian Res 5:33

Zhang H, Zhang G-F, He Z-Y, Li Z-Y, Zhang G-X (2014) Prospective evaluation of 3t MRI findings for primary adnexal lesions and comparison with the final histological diagnosis. Arch Gynecol Obstet 289(2):357-364

\section{Submit your manuscript to a SpringerOpen ${ }^{\circ}$ journal and benefit from:}

- Convenient online submission

- Rigorous peer review

- Immediate publication on acceptance

- Open access: articles freely available online

- High visibility within the field

- Retaining the copyright to your article

Submit your next manuscript at $\boldsymbol{\nabla}$ springeropen.com 